\title{
Mercure.
}

Il résulte de l'étude succincte d'une vingtaine de dessins de cette planète, montrant de nombreux détails, et pris au Massegros durant l'élongation de septembre, que la période de rotation de Mercure coincide avec la période de révolution.

Le 25 octobre 1909 .

R. Farry-Desloges.

\section{Ein neues Observatorium}

wurde in Nischburg, Böhmen, errichtet. Dasselbe hat als Hauptarbeitsgebiet die Astrophotographie und die Untersuchungen über den Lichtwechsel veränderlicher Sterne, und ist mit einem I $35 \mathrm{~mm}$ Merz-Refraktor und einem photographischen Doppelrefraktor $(1.65 \mathrm{~m}$ und $135 \mathrm{~mm}$ photogr., $135 \mathrm{~mm}$ visuell) ausgerüstet, zu welchen in späterer Zeit ein $300 \mathrm{~mm}$ Refraktor hinzutreten wird. Fin photochemisches

Observatorium Nischburg, I 909 Okt. 25.
Laboratorium ist der Sternwarte angeschlossen, so daß dieselbe nach der Fertigstellung des Neubaues an allen Arbeiten ihres Gebietes wird teilnehmen können. Der Unterzeichnete bittet die Institute um Unterstützung der neuen Arbeitsstätte der Astronomie durch Zusendungen der Publikationen im Austausch gegen ihre eigenen Veröffentlichungen, welche demnächst zur Versendung gelangen werden.

\section{Bekanntmachung.}

Auf das Preisausschreiben der Astronomischen Gesellschaft (siehe V. J. S. Jahrgang 39, Seite 149, A. N. No. 3977) sind rechtzeitig zwei Einsendungen erfolgt. Die erste mit dem Motto "Quod potui feci " mußte außer Betracht bleiben, da das Wesentliche ihres Inhaltes schon vorher publiziert worden ist.

Die zweite mit dem Motto "Isti mirantur stellam《 enthält nicht nur eine vollständige Lösung der gestellten Aufgabe, sondern in mancher Richtung noch mehr, als in der Formulierung der Preisaufgabe verlangt wird.

Der Vorstand der Astronomischen Gesellschaft hat deshalb einstimmig beschlossen, der genannten Arbeit den vollen Preis zuzuerkennen. Die Eröffnung des beigelegten Kuverts ergab die Namen der Autoren:

\section{P. H. Coucll und Andreu C. D. Crommolin.}

München, Berlin, Potsdam, 23. Oktober 1909.

H. Secliger, Vorsitzender. R. Lehmann-Filhes, G. Miller, Schriftführer.

\section{Literarische Anzeige.}

A. Müller, S. J. Galileo Galilei und das kopernikanische Weltsystem. Der (Yalilei-Prozeß $\left(163_{2}-1633\right)$ nach Ursprung, Verlauf und Folgen.

184 bezw. 205 Seiten. Freiburg i. Br., Herdersche Verlagshandlung 1909.

Die beiden Schriften bilden eine zusammenhängende Biographie des großen italienischen Naturforschers, die der Verfasser seinen bekannten Lebensskizzen der anderen beiden der neueren Astronomie die IVege ebnenden Meister, Kopernikus und Keppler, hinzufügt. Die Darstellung der für die Wissenschaft wichtigsten Momente des bewegten Lebensganges Galileis und die Schilderung des an die Veröffentlichung seines glänzenden "Dialogs ïber die Weltsysteme« sich knüpfenden und zur Verurteilung wegen des Verdachtes der Häresie führenden Inquisitionsprozesses erfolgt an der Hand der jetzt in der neuen unter dem Patronate des Königs von Italien veranstalteten Herausgabe der gesamten Schriften Galileis, so daß der Leser in der Iage ist, sich ein selbständiges abschließendes Urteil unabhängig von den Ausführungen des Verfassers zu bilden.

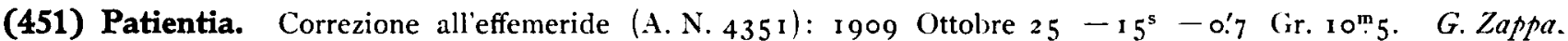

Anzeige. An der Treptow-Sternwarte ist die Stellung eines wissenschaftlichen Assistenten zu besetzen. Meldungen unter Einsendung des Lebenslaufes und Studienganges an die Direktion der Treptow-Sternwarte erbeten.

Kaufgesuch. Durch Vermittlung der Redaktion wird eine zu einem 7- oder 8-zölligen Refraktor passende Montierung zu erwerben gesucht.

Inhalt zu Nr. 4366. G. Eberhard und H. Lutendorff. Zur Reduktion von Sternspektrogrammen. 36r. - W. Miinch. Photometrische Beobachtungen einiger vom Kometen $1908 \mathrm{c}$ (Morehouse) bedeckten Sterne. $36_{3} . \quad$ S. Kostinsky. Über die Eigenbewegung der Sterne in der lingebung der Sternhaufen $\chi$ und $h$ Persei. 369. - P. H. Collell. Three new planets. 37ı. - M. Wolf. Photographische Aufnahmen von kleinen Planeten. 373. - F. H. Metcalf. Observations of Phoebe and asteroids. 373. G. Lecointe. Halleyscher Komet 1909 c. 373. - F.Porro. Auffindung des Winneckeschen Kometen 19o9 d. 373. - R. FarryDesloges. Mercure. 375. - L. Pracka. Ein neues Observatorium. 375., - Bekanntmachung. 375 . - Literarische Anzeige. 375. - Kleine Mitteilungen. 375. 